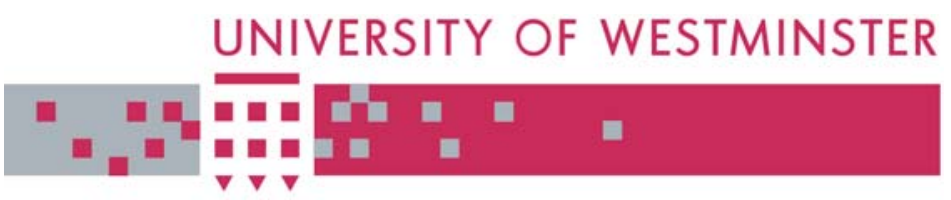

\title{
WestminsterResearch
}

http://www.wmin.ac.uk/westminsterresearch

\section{Turbo-code division multiple access: capacity enhancement of mobile satellite systems using narrowband multiuser detection.}

\author{
Panos Fines ${ }^{1}$ \\ Paul Febvre ${ }^{2}$ \\ Ekaterina Christofylaki ${ }^{3}$ \\ ${ }^{1}$ Wireless Intelligent Systems Ltd., London \\ 2 Inmarsat Ltd., London \\ ${ }^{3}$ School of Informatics, University of Westminster
}

Copyright (C) [2008] IEEE. Reprinted from the 10th International Workshop on Signal Processing for Space Communications (ESA): Proceedings of a meeting held 6-8 October 2008, Rhode Island, Greece. IEEE, pp. 1-5. ISBN 9781424425723.

This material is posted here with permission of the IEEE. Such permission of the IEEE does not in any way imply IEEE endorsement of any of the University of Westminster's products or services. Personal use of this material is permitted. However, permission to reprint/republish this material for advertising or promotional purposes or for creating new collective works for resale or redistribution to servers or lists, or to reuse any copyrighted component of this work in other works must be obtained from the IEEE. By choosing to view this document, you agree to all provisions of the copyright laws protecting it.

The WestminsterResearch online digital archive at the University of Westminster aims to make the research output of the University available to a wider audience. Copyright and Moral Rights remain with the authors and/or copyright owners.

Users are permitted to download and/or print one copy for non-commercial private study or research. Further distribution and any use of material from within this archive for profit-making enterprises or for commercial gain is strictly forbidden.

Whilst further distribution of specific materials from within this archive is forbidden, you may freely distribute the URL of the University of Westminster Eprints (http://www.wmin.ac.uk/westminsterresearch).

In case of abuse or copyright appearing without permission e-mail wattsn@wmin.ac.uk. 


\section{Turbo-Code Division Multiple Access:}

\section{Capacity Enhancement of Mobile Satellite Systems Using Narrowband Multiuser Detection}

\author{
P. Fines \\ Wireless Intelligent Systems Ltd., \\ London, U.K. \\ panos_fines@btinternet.com
}

E. Christofylaki

School of Informatics, University of Westminster, London, U.K.

ekateric@wmin.ac.uk

\author{
P. Febvre \\ Inmarsat Ltd. \\ London, U.K. \\ paul_febvre@inmarsat.com
}

\begin{abstract}
This paper presents a new type of multiple access for fixed and mobile satellite communication systems aiming at improving the bandwidth efficiency of highly reliable links suited for air traffic management and other safety services, using compact and low cost terminals. The purpose of this work is to incorporate highly redundant turbo coding technology in the air interface and present a novel receiver architecture using narrowband multiuser detection to enhance the performance of the reservation access and random access signaling.
\end{abstract}

Keywords - turbo-code division multiple access, multiuser detection, mobile satellite satellite aeronautical communications, air traffic management, safety services.

\section{INTRODUCTION}

The operational cost of low data rate mobile geostationary (GEO) satellite systems is relatively expensive when compared to fixed or transportable counterparts due to their low gain antenna, limited transmit power and extensive fading margin. For such systems more satellite resources, such as bandwidth and power, should be made available to accommodate for the poor propagation conditions which usually become worse as the user terminals operate at the edge of coverage viewing the satellite at lower elevation angle (e.g. as low as $5^{\circ}$ ). One approach is to limit the service area so that only high elevation satellite links are allowed (e.g. higher than $20^{\circ}$ ) ensuring good propagation conditions, but this clearly does not satisfy the requirements for provision of services at high latitudes such as Northern European countries. One solution is to use higher gain antennas at the terminals and to include some mechanism to steer the antenna beam towards the satellite. Another solution is to design waveforms with time-diversity to provide protection against short-duration deep multipath fades but this has the disadvantage that low-latency services such as voice cannot be supported. A further solution that has not yet been fully exploited is the concept of space-diversity antenna subsystems, but while this may be suitable for some types of mobile installations the possible applications are somewhat limited.

An example of a modern mobile satellite system is the Inmarsat Broadband Global Access Network (BGAN) which has been deployed to provide high speed IP data services to very compact transportable terminals using a global network of multi-beam GEO satellites at L-band. Recently, the BGAN system has been enhanced to extend the mobile terminal portfolio to include land mobile, maritime and aeronautical services such as Swiftbroadband (SBB). Inmarsat is continuously enhancing the BGAN system to support new services such as push-to-talk netted voice-over-IP and other interactive group data applications. Such applications involve bursty traffic from user to user interaction with very tight latency requirements. The low-latency of bursty traffic requirement is difficult to address efficiently using a conventional TDMA waveform such as employed in the existing BGAN air interface. New radio resource management algorithms, including modifications to the signaling and the behavior of both Mobile Terminals and Satellite Access Stations are likely to be introduced to support such service capabilities in the near future. However, it has been recognized that the introduction of extensions to the existing BGAN physical layer able to support unscheduled asynchronous transmissions from Mobile Terminals (MT) in the return link, will both minimize system complexity while enhancing system performance and capacity for such applications.

An example of mobile satellite service employing compact low gain antennas and requiring high reliability over the widest possible area is air traffic management (ATM). The forecast increase in air traffic has stimulated research into advanced satellite based systems for air traffic management. Satellitebased systems are already used for provision of aeronautical safety services in Oceanic airspace, and offer significant cost 
advantages as a secondary communications path to improve overall system availability for Continental airspace, but systems employing low gain antennas on the aircraft to provide higher data rates are still to be defined and the expected performance remains to be validated in an operational environment. Current systems for ATM in Oceanic airspace such as Inmarsat Aeronautical Mobile Satellite Service (AMSS) and the Japanese MTSAT employ mostly intermediate and high gain antennas which are large and expensive when small civil aircrafts are considered. Some of the work presented in this paper was conducted in a project co-funded by the European Commission within the Sixth Framework Programme: Airborne New and Advanced Satellite techniques and Technologies in A System Integrated Approach (ANASTASIA). The activities of ANASTASIA range from the elaboration of operational needs to system simulations and trials with validated avionics architecture and key technologies. Under this context, the techniques presented in this paper were considered as potential future enhancements of the SBB system return link. The forward SBB link design concepts are different and potential enhancements are presented in [5].

\section{NEW TYPE OF MULTIPLE ACCESS SCHEME}

State-of-the art satellite systems (e.g. Inmarsat BGAN, DVBS/RCS, etc) use allocation schemes which operate in both frequency and time domains (e.g. MF-TDMA) as shown in Figure 1 , to simplify the transmission, reception and operation of multiple terminal access within the same frequency band. This, however, puts organizational and computational burden on the radio network controller. The principle concern with an MF-TDMA scheme is that the energy density in the channel is largely determined by the performance of the worst-case mobile terminals that are sharing a particular communications channel at any time, as the worst case terminal requires the highest percentage of time on the channel in order to provide a minimum quality of service. Joint access of spectrum as shown in Figure 2 together with multi-burst decoding at the receiver end, has the potential of significantly increasing the system capacity by increasing the energy density in the channel, while simultaneously improving service latency and reducing the overheads associated with network control functions. Complexity is transferred from the signaling and control infrastructure into the receiver, this being equipped with enhanced signal processing techniques making possible to extract information streams from composite signals.

The purpose of this work is to evaluate the use of highredundancy turbo-coding technology in the return direction and the associated novel receiver architectures which may be used to enhance the performance of the reserved and random-access signaling.

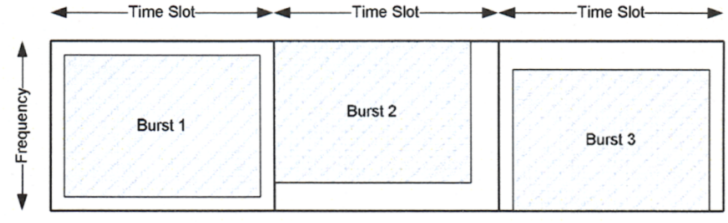

Figure 1. Conventional Time-Division Multiple Access Scheme

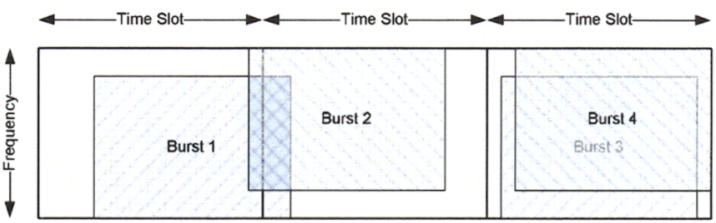

Figure 2. Joint Access Multiple Access Scheme with Multi-burst Receiver

In contrast to the current BGAN return link, unscheduled transmissions from vehicular, maritime and aeronautical including helicopter terminals reuse the satellite resources. All these terminals use the same time and frequency slot for their transmission. The new type of Satellite Access Station (SAS) receiver processes the received composite signal from all these transmissions which are corrupted by high mutual interference and detects each transmission. Therefore, the satellite spectrum is not shared but instead is reused by a number of terminals and this feature increases dramatically the system capacity, reduces the system latency and simplifies the signaling protocols.

Traditionally, a similar multiple access scheme is implemented using DS-CDMA technologies. However, the proposed BGAN system enhancement uses highly redundant turbo coded narrowband transmissions instead of CDMA techniques and their associated spreading sequences. For this reason the proposed multiple access scheme is named Turbo Code Division Multiple Access scheme (TCDMA) and uses narrowband multiuser detection. The TCDMA signals in space are totally compatible with the legacy BGAN system and at the same time are extremely robust against multipath fading from different mobile satellite propagation channels and interference, originating from similar transmissions which share simultaneously the same frequency channel. In contrast to the traditional CDMA, the capacity of a TCDMA system increases as the simultaneous transmissions differ in power. Therefore, there is no need for power control signaling. Small time and frequency errors between the simultaneous transmissions and multipath fading are different for each MT helping the receiver to discriminate the various transmissions. The system capacity increases as the coding rate decreases and the transmissions become uncoordinated. This is a TCDMA significant feature in contrast to traditional approaches [1]. Another attractive feature is that robustness of each transmission increases as the traffic loading decreases and this is provided without any involvement from signaling protocols. Therefore, the proposed TCDMA multiple access scheme is very suitable for sporadic unscheduled traffic from MT equipped with low gain antennas. Some of the benefits of TCDMA configuration are: 
Enhanced random access capacity: Minimizes signaling delay, maximizes capacity for random access and simplifies protocols for sporadic transmissions

Enhanced user traffic capacity: Scheduled and unscheduled user traffic can share return slots

Reduction of signaling latency: The signaling protocols introduce considerable latency due to the satellite round trip

Simplification of network protocols: Since slots can be shared, less radio resource reservation and signaling are necessary

However, the benefits are not for free: the complexity of a multi-burst receiver is significantly higher than that of a singlechannel receiver. Complexity is transferred into the receiver incorporating new functions which can extract information from a composite signal. This may not be a trivial task since the complexity of a multi-burst receiver is higher than conventional single-burst receivers. In general, as the receiver becomes more complex, its performance improves. However, there is a practical limit on how complex the receiver can be and the approach taken in this study is to derive a minimum complexity architecture that retains most of the performance benefits mentioned above. The architecture used in this study appears to bridge the gap between single burst and multi-burst receivers. Furthermore, this architecture appears to perform very well with a realistic operational scenario and with the potential of approaching the theoretical capacity of the multiple access channel.

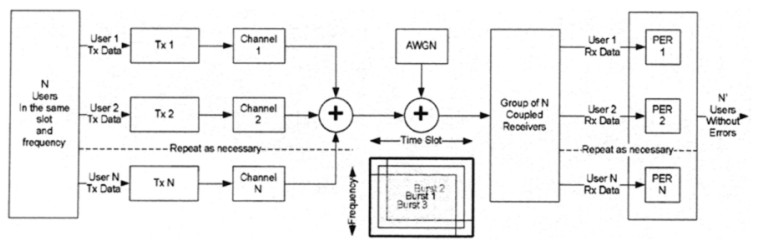

Figure 3. TCDMA System Model

\section{TCDMA SYSTEM MODEL}

The system model is shown in Figure 3. There are up to $\mathrm{N}$ users, each utilizing a transmitter for sending data. The radio resource management system allocates a single time/frequency slot for all these users to access the channel in random on demand fashion. All $\mathrm{N}$ users are transmitting simultaneously in the slot. Each transmission travels via a different propagation channel which introduces different delay, attenuation, frequency shift, multipath fading and phase noise. The proposed system has no specific means for ensuring signal orthogonality. However, it should be noted that each channel introduces some unique signature per user by affecting at least some (if not all) of the previously mentioned signal parameters.

All the traffic bursts have coding rates from $R=1 / 3$ down to $\mathrm{R}=1 / 9$ and are generated by a parallel concatenated turbo encoder which is an extension of the CCSDS standard [2]. The turbo interleaver is as specified for the BGAN air interface and it is an improved version of the CCSDS standard optimized for small packet sizes [4]. The turbo code performance depends on the coding rate and the data size and for $R=1 / 5$ and 504 data bits its AWGN performance is shown in Figure 4. In the newly defined BGAN bursts the FEC symbols are interleaved with symbols known (UW) at the receiver end. The receiver uses the UW symbols as pilots for demodulating correctly each burst.

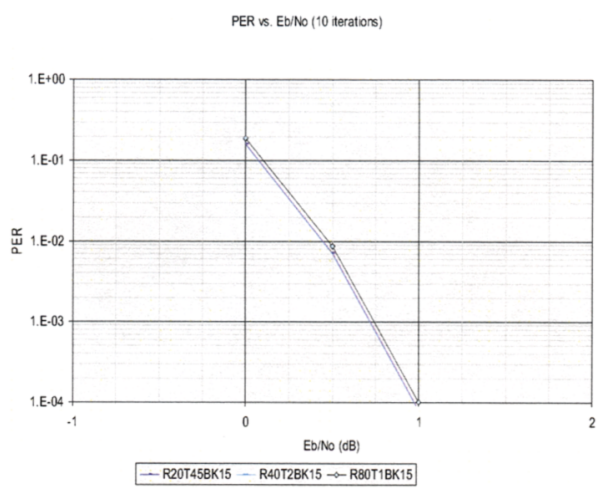

Figure 4. Turbo code performance

At the receiving end, a normal BGAN receiver for a single user shall fail to recover the user data since the bursts interfere heavily with each other. In this study we investigate the performance of a group of $\mathrm{N}$ receivers which exchange information in order to recover all bursts simultaneously as shown in Figure 5. Each user link is characterized by an individual Packet Error Rate (PER) counter. In every slot, there is a counter which measures how many user bursts have been received without errors ( $\mathrm{N}$ users transmit and $\mathrm{N}^{\prime}$ are received without errors). The statistical mean of $N^{\prime}$ is an indication of the receiver effectiveness assuming the type of multiple-access described above. With an ideal receiver $\mathrm{N}^{\prime}=\mathrm{N}$ for $\mathrm{N}=1,2, \ldots$ In practice there must be a threshold beyond which $N^{\prime}<N$ and $N^{\prime}$ approaches zero as $\mathrm{N}$ increases. The ratio $\mathrm{N}^{\prime} / \mathrm{N}$ is the effective throughput indicating the average number of successfully decoded bursts. Of course, when $N$ ' Mobile Terminals are decoded there is an average PER associated with each MT.

Due to the unscheduled nature of return transmissions and the satellite long round trip, transmission coordination in terms of timing, frequency and power may not be possible. Therefore, another requirement is that the multi-burst system should operate without the need of accurate power control. Timing and frequency may be estimated accurately since the MT is equipped with GPS. However, the proposed system capacity may be increased if the transmissions are not employing accurate timing, frequency and power. Of course, the bursts should always fall within the time slot and frequency boundaries for avoiding adjacent channel interference and their power should limited so that extensive co-channel interference is also avoided in nearby spot beams. The propagation channel parameters contain random elements that are unique for each burst and could improve the overall capacity. On the other hand, accurate estimation of small disturbances may make the demodulator's job more difficult and performance loss may be experienced. 


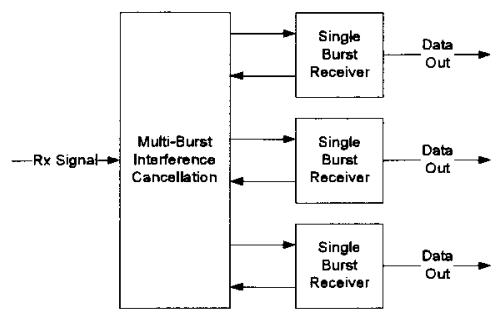

Figure 5. TCDMA Multi-burst Receiver

\section{TCDMA PERFORMANCE RESULTS}

The results in this section assume $20 \mathrm{~ms}$ bursts with rate $1 / 3$ turbo coding. The receiver throughput is presented in Figure 6 where all bursts have random power, time and frequency within the following ranges:

a) The $\mathrm{Eb} / \mathrm{No}$ is random between 8 and $13 \mathrm{~dB}$ plus Rician fading with carrier to multipath power ratio $\mathrm{C} / \mathrm{M}=10 \mathrm{~dB}$ and fading bandwidth $\mathrm{Fd}=20 \mathrm{~Hz}$.

b) Timing uncertainty: uniform random $3 \mathrm{~ms}$

c) Frequency uncertainty $\pm 200 \mathrm{~Hz}$

With a single burst receiver, the maximum throughput is of course 1 burst. When two bursts arrive in a single slot processed by a conventional single burst receiver, about a quarter of them are detected correctly on average. This is not a surprise since the fading occasionally produces great energy difference between the two bursts and the strongest one can be decoded correctly. On the other hand, using a multi-burst receiver the average maximum throughput is 3 bursts. The average PER is equivalent to the burst error rate shown in Figure 7. The PER curve indicates that the PER increases rapidly above 2 bursts per slot.

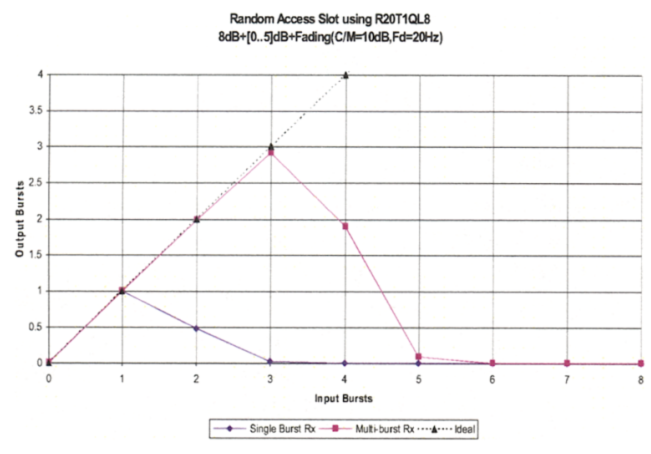

Figure 6. Throughput example with rate $1 / 3$ code

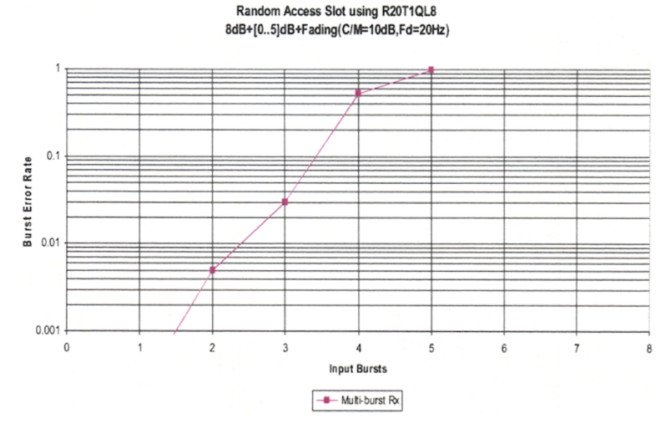

Figure 7. Packet Error Rate example with $1 / 3$ code

Of course, the implementation of each receiver type is not a trivial issue. Indeed, the multi-burst receiver is possibly among the most complicated receiving systems. The proposed receiver architecture is a trade-off between complexity and performance aiming at a 'reasonable' complexity solution

Further results are presented assuming $80 \mathrm{~ms}$ bursts with rate $1 / 5$ turbo coding and three types of receiver:

The first type uses the UW for channel state estimation and demodulation (UW Rx). This has moderate complexity and it is very similar to the conventional receiver.

The second type uses a new type of iterative receiver (Turbo Rx) in which the demodulator and FEC decoder iterate exchanging information [3]. This receiver provides near optimum performance at the expense of higher complexity which scales according to channel conditions.

Finally, the third type employs a perfect demodulator (Perfect Rx). This is not practically realizable but sets the benchmark against which the implementation loss of the other two types mentioned above can be measured.

The receiver throughput is presented in Figure 8 where all bursts have random power, time and frequency within the following ranges:

a) $\mathrm{Eb} / \mathrm{No}$ random in the range between 10 and $15 \mathrm{~dB}$ plus Rician fading with $\mathrm{C} / \mathrm{M}=10 \mathrm{~dB}$ and fading bandwidth $\mathrm{Fd}=20 \mathrm{~Hz}$.

b) Timing uncertainty: uniform random $3 \mathrm{~ms}$

c) Frequency uncertainty $\pm 200 \mathrm{~Hz}$

It should be noted that the available Eb/No is extensive and well above the fading margin required. However, this is an example of how one can trade power for capacity.

Using a multi-burst receiver the average throughput is shown in Figure 8. The maximum throughput is 9 bursts achieved with an ideal receiver. The average PER is shown in Figure 9. The PER curves indicate that the PER of the UW Rx degrades rapidly as the number of bursts increase to more than 3. On the other hand, the Turbo $\mathrm{Rx}$ approximates the performance of the Perfect $\mathrm{Rx}$. The UW Rx achieves remarkably good performance considering its low complexity. 
However, the Turbo Rx shows how well it can approximate the Perfect Rx. Since the complexity of the Turbo Rx is scalable, one could trade performance for complexity reduction.

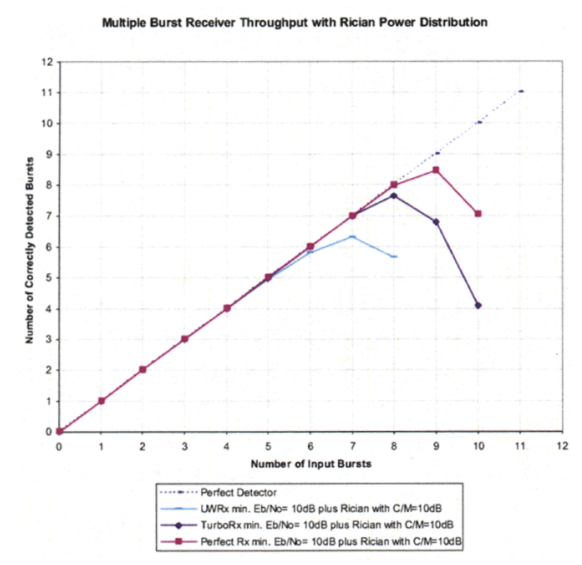

Figure 8. Throughput example with rate $1 / 5$ code

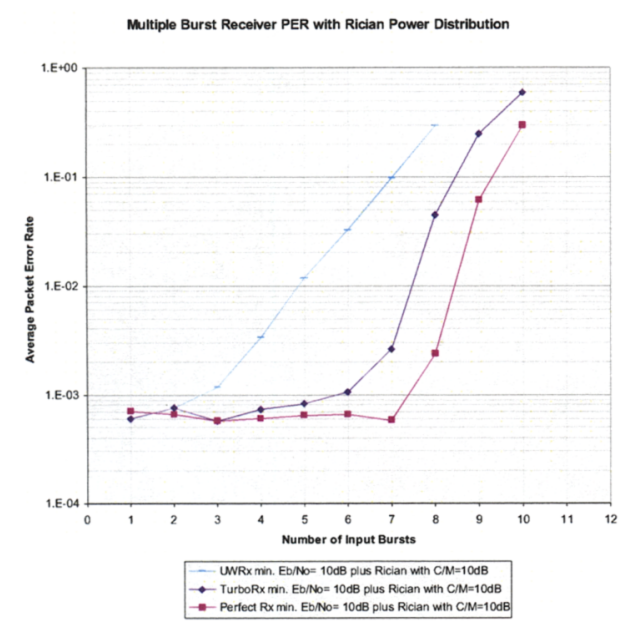

Figure 9. Packet error rate example with rate $1 / 5$ code

\section{CONCULSIONS}

State-of-the art fixed (e.g. DVBS2/RCS) or mobile satellite systems such as the Broadband Global Access Network (BGAN) and its aeronautical version Swiftbroadband system (SBB) use mostly orthogonal allocation schemes in frequency and time to simplify the transmission and reception technologies. The SBB return link is a typical example, where each aeronautical terminal transmits a burst of data always within unique time/frequency slot. This, however, puts organizational and computational burden on the network controller.

This paper presents a new type of multiple access which improves the bandwidth efficiency and reduces the latency of modern satellite systems using highly redundant turbo coding and new receiver architecture. It considers a multi-burst receiver with a multiuser detector at the satellite access station capable of detecting more than one burst per time and frequency slot. The proposed scheme uses highly redundant turbo coded narrowband transmissions. For this reason the proposed multiple access scheme is named Turbo Code Division Multiple Access scheme (TCDMA).

Joint accessing of spectrum using multiple-burst transmission and multiuser decoding strategies has the potential of significantly increasing the system capacity while reducing the overhead associated with network control functions and various signaling latencies. Complexity is transferred into the receiver, equipped with signal processing functions which can extract information streams from composite signals. With the rapid advances of VLSI technology multi-burst reception appears within reach of modern VLSI chips.

The multi-burst receiver includes several sub-receivers and sub-decoders all operating several times until successful detection of the incoming bursts is achieved. The results reveal that the proposed technology nearly doubles the throughput over the conventional single burst receiver. In the random access mode, the application of multi-burst receivers may also eliminate collisions which in turn, shall reduce the signaling latency of mobile terminals requesting system resources.

Clearly, there is a significant increase in receiver complexity but the benefit is also very significant: the system access capacity increases with the number of successfully detected bursts. The main question is if the system advantages provided by the multi-burst receivers shall outperform their computational complexity requirements and their associated cost.

\section{REFERENCES}

[1] C. Schegel and A. Grant, "Coordinated Multiuser Commuincations", 2006, Springer.

[2] TM Synchronization and Channel Coding, CCSDS 131.0 B-1, September 2003.

[3] J. Hamkins \& D. Divsalar, "Coupled Receiver/Decoder for Low Rate Turbo Codes", ISIT, Yokohama, Japan, 2003.

[4] P. Fines, E. Christofylaki, S. Papaharalabos and P. Febvre, "Low Rate Turbo Code Extensions and Modem Design for High Reliability Satellite Links", ESA TTC 2007

[5] P. Fines and E. Christofylaki, "Advanced Satellite Technologies for Air Traffic Management”, ASMS'2008, Bologna 